Revue internationale P.M.E.

Économie et gestion de la petite et moyenne entreprise

\title{
Le développement des avantages compétitifs dans les PME
}

\section{Ingolf Bamberger}

Volume 1, numéro 1, 1988

URI : https://id.erudit.org/iderudit/1007872ar

DOI : https://doi.org/10.7202/1007872ar

Aller au sommaire du numéro

Éditeur(s)

Presses de l’Université du Québec

ISSN

0776-5436 (imprimé)

1918-9699 (numérique)

Découvrir la revue

Citer cet article

Bamberger, I. (1988). Le développement des avantages compétitifs dans les PME. Revue internationale P.M.E., 1(1), 23-41. https://doi.org/10.7202/1007872ar

\section{Résumé de l'article}

Selon l'auteur, de manière théorique, les avantages compétitifs sont déterminés par cinq complexes de variables (= conditions externes et internes de l'entreprise) : les caractéristiques des industries et des marchés, les ressources internes de l'entreprise, les objectifs poursuivis, la personnalité des décideurs stratégiques et les stratégies produits/marchés. Une enquête en1985, dans huit pays européens, auprès de 1135 PME, issues de trois branches (habillement, industrie agro-alimentaire et électronique) a révélé que les PME utilisent les six facteurs suivants pour élaborer des avantages compétitifs sur leurs marchés : compétence et réputation, potentialité de marketing, compétence technologique et service, conditions financières, créativité et différenciation des produits, coûts bas et politique des prix. Le premier facteur (compétence et réputation) regroupe les items qui peuvent être considérés comme des facteurs de base. L'importance des cinq autres facteurs et de leurs éléments respectifs varie beaucoup plus en fonction des conditions externes et internes de l'entreprise.
Tous droits réservés Presses de l’Université du Québec, 1988
Ce document est protégé par la loi sur le droit d'auteur. L'utilisation des services d'Érudit (y compris la reproduction) est assujettie à sa politique d'utilisation que vous pouvez consulter en ligne.

https://apropos.erudit.org/fr/usagers/politique-dutilisation/ 


\title{
Le développement des avantages compétitifs dans les PME*
}

\author{
Ingolf BAMBERGER \\ Directeur du Laboratoire Stratégie et Structure \\ Université de Rennes
}

\begin{abstract}
RESUME
Selon l'auteur, de manière théorique, les avantages compétitifs sont déterminés par cinq complexes de variables (= conditions externes et internes de l'entreprise) : les caractéristiques des industries et des marchés, les ressources internes de l'entreprise, les objectifs poursuivis, la personnalité des décideurs stratégiques et les stratégies produits/marchés. Une enquête en 1985, dans huit pays européens, auprès de 1135 PME, issues de trois branches (habillement, industrie agro-alimentaire et électronique) a révélé que les PME utilisent les six facteurs suivants pour élaborer des avantages compétitifs sur leurs marchés : compétence et réputation, potentialité de marketing, compétence technologique et service, conditions financières, créativité et différenciation des produits, coûts bas et politique des prix. Le premier facteur (compétence et réputation) regroupe les items qui peuvent être considérés comme des facteurs de base. L'importance des cinq autres facteurs et de leurs éléments respectifs varie beaucoup plus en fonction des conditions externes et internes de l'entreprise.
\end{abstract}

\section{ABSTRACT}

According to the author, the concurrential advantages are, theoretically, determined by 5 groups of variables (= external and internal firm conditions) which are : industry features, internal firm resources, wanted goals, the personality of the strategic responsibles and the products / Market strategies. In 1985, a study was made in 8 European countries, among 1135 firms of small and middle scale and coming from three different sectors (Clothing, Agricultural and Food industries, and Electronics). That study showed that companies of small and middle scale use six factors to work out concurrential advantages on markets. These factors are the following ones : skill and fame, marketing potentiality, service and technological skill, financial conditions, creativity and products differentiation and low costs and policy prices. The first factor, that is to say skill and fame, includes the items which can be considered as main factors. The significance of the 5 other factors and their respective elements changes much more according to external and internal firm conditions.

* Cette enquête a été réalisée avec le soutien de la Fondation Nationale pour l'enseignement de la Gestion des Entreprises (F.N.E.G.E.). L'auteur exprime ses remerciements profonds à la Fondation. 


\section{RESUMEN}

Según el autor, las ventajas competitivas están, teóricamente, determinadas poe cinco conjuntos de variables (: condiciones externas e internas de la empresa) : las características de la industrías y de los mercados, los recursos internos de la empresa, los objectivos descados, la personalidad de los responsables estratégicos y las estrategias productos/ mercados. En 1985, una encuesta fue realizada, en ocho paises europeos, entre 1135 PME procedentes de tres sectores (confección, industría agroalimenticia y electrónica). Esta encuesta endicó que las PME utilizan seis factores para elaborar ventajas competitivas en sus mercados. Los seis factores son los siguientes : competencia y fama, potencialidad de marketing (comercialización), competencia tecnológica y servicio, condiciones financieras, creatividad y diferenciación de los productos, costes bajos y política de precios. El primer factor (es decir competencia y Fama) constá de los «items" que pueden ser considerados como factores fundamentales. La importancia de los cinco otros factores y de sus elementos respectivos cambia mucho màs en función de condiciones externas e internas de la empresa. 


\section{Introduction}

L'élaboration des avantages compétitifs est un des composants principaux du comportement stratégique. Leurs bases et leur élaboration font actuellement l'objet d'une discussion intense dans la théorie stratégique. Cette discussion est la conséquence de l'intérêt pour les stratégies de domaine et, notamment, les stratégies compétitives. Elle a été fortement promue par Porter ${ }^{1}$ dans des publications récentes.

L'objet de ce papier est une analyse théorique et empirique des compétences utilisées par les P.M.E. pour développer des avantages compétitifs sur leurs marchés, et leurs déterminants.

\subsection{Les avantages compétitlifs}

Le concept de l'avantage compétitif n'est pas nouveau. Il est traditionnellement un des concepts clé en marketing et en sciences économiques. C'est un concept qui est inné à la concurrence. Une entreprise qui souhaite survivre et réussir sur un marché, devrait posséder certaines caractéristiques distinctes, devrait avoir un avantage unique par rapport à ses concurrents. Un avantage compétitif peut ainsi être défini comme une position unique qu'une entreprise développe vis-à-vis de ses concurrents (Hofer et Schendel, 1978).

Les avantages compétitifs se fondent sur l'élaboration de ressources et/ou des décisions sur l'étendue des activités. Pour créer et maintenir des avantages compétitifs, l'entreprise développe des ressources et aptitudes spécifiques, appelées des compétences distinctives. De cette façon, il est utile de distinguer les avantages positionnels, c'est-à-dire les avantages compétitifs, des sources de ces avantages, les compétences distinctives.

Le but du développement des avantages compétitifs est de créer une potentialité de bénéfices pour l'entreprise. Cependant, l'existence de différences de ressources par rapport aux concurrents n'aboutit pas automatiquement à un avantage compétitif et une (durable) potentialité de bénéfices. Des différences dans le déploiement des ressources et la définition de l'étendue des activités ne deviennent des avantages compétitifs et ne créent des potentialités de bénéfices que si :

- les différences créent de l'utilité pour un groupe de client suffisamment grand ;

- les clients sont prêts à payer pour cette utilité et

- les avantages peuvent être défendus sur une période assez longue.

Les avantages compétitifs sont développés par la création de valeur, de l'utilité pour les clients.

1 Cf. Porter (1985) (1980); mais également Rothschild (1984); Day (1984); Pümpin (1983); Henderson (1983). 


\subsection{Types d'avantages compétitifs}

Correspondant aux différentes manières de créer de la valeur pour les clients, le nombre de types d'avantages compétitifs possibles est pratiquement illimité. Les avantages compétitifs peuvent être des coûts et des prix plus bas, des délais de livraison plus courts, une gamme de produits très large, la qualité, une bonne image de marque ou une supériorité technologique.

Suite à la conception de Porter $(1980,1985)$, on utilise aujourd'hui en général la classification suivante :

- domination par les coûts, c'est-à-dire le développement des avantagescompétitifs par des coûts et des prix bas ;

- la différenciation, par exemple par la qualité, la politique de marque ou un service particulier et

- leur combinaison avec la largeur des activités stratégiques, la focalisation, la concentration sur des segments spécifiques du marché.

Dans ce contexte, on pense que la poursuite d'une domination par les coûts et d'une différenciation est, en général, conflictuelle dans la mesure où une différenciation tend à augmenter les coûts.

\subsection{L'élaboration des avantages compétitifs}

L'élaboration des avantages compétitifs aétél'objet de plusieurs publications récentes (Day, 1985, Porter, 1985, Rothschild, 1984). L'accent est mis notamment sur les différents types d'avantages compétitifs, leurs sources ainsi que, dans une perspective normative, les activités pour les mettre en oeuvre. Dans ce contexte, on peut notamment mentionner l'approche de Porter introduisant la conception très stimulante de la chaîne de création de valeur ("Value chain»).

On a, cependant, négligé l'analyse des facteurs qui déterminent quels types d'avantages compétitifs sont développés. Cette question fait l'objet de cette étude. Il sera analysé, dans une perspective descriptive et empirique :

- quels facteurs sont utilisés par des petites et moyennes entreprises pour obtenir sur leurs marchés une position concurrentielle favorable et, en particulier,

- quels sont les déterminants influant sur les types d'avantages compétitifs élaborés.

L'étude empirique du développement des avantages compétitifs dans les P.M.E. se fera sur la base d'un cadre de référence théorique décrivant les relations entre les avantages compétitifs et leurs déterminants potentiels. Nous pensons que ce cadre de référence est d'une nature générale et peut, de cette façon, être utilisé aussi bien par rapport aux petites que par rapport aux grandes entreprises. Bien entendu, les conditions spécifiques relatives aux différentes variables diffèrent d'une entreprise à l'autre. 


\section{Le cadre de référence théorique}

L'analyse des déterminants des avantages compétitifs est effectuée dans deux perspectives : une perspective théorique et une perspective pratique. Dans une perspective théorique, on étudiera les caractéristiques externes et internes expliquant les différences dans l'utilisation des avantages compétitifs entre les entreprises. Dans une perspective pratique, le cadre de référence décrira les facteurs dont une entreprise devrait tenir compte quand elle prend ses choix stratégiques par rapportà l'élaboration de sa position compétitive.

On suppose que les avantages compétitifs sont déterminés par cinq complexes de variables (figure 1) : les caractéristiques des industries et des marchés, les ressources internes de l'entreprise, les objectifs poursuivis, la personnalité des décideurs stratégiques et les stratégies produits/marchés. Ces cinq complexes de variables, les avantages compétitifs et leur mise en oeuvre ainsi que la performance de l'entreprise sont fortement interdépendants. Il est difficile d'établir des relations causales.

\subsection{Les caractéristiques de l'industrie et du marché}

Un premier déterminant, fort influant, est constitué par les conditions de l'industrie et du marché. Elles forment le contexte structurel direct pour la concurrence et incluent, notamment, les attributs de la demande et de la structure de l'industrie.

En ce qui concerne la demande, les types de besoins des clients, la sensibilité de la demande au prix, l'homo ou l'hétérogénéité, le volume, les liaisons entre les achats, la stabilité, la fréquence d'achat et les expériences sont d'un intérêt particulier :

- le type de besoins des clients se réfêre aux attributs du produit ou du service qu'un client apprécie, comme par exemple la qualité, une livraison rapide, une assistance technique et le prestige. Ils trouvent leur expression dans la chaine de création de valeur du client et de ses critères d'achat.

- L'hétérogénéité de la demande est la condition nécessaire pour la segmentation et la poursuite d'une stratégie de différenciation.

- L'élasticité de la demande au prix détermine, ensemble, avec son volume et son homogénéité, l'importance d'une stratégie de coûts et de prix bas.

- Les liaisons entre les achats ainsi que les expériences des acheteurs peuvent avoir une influence sur la largeur de la gamme de produits.

- La stabilité de la demande est importante par rapport à la flexibilité, la fréquence d'achat par rapport au système de distribution et l'image de marque du producteur.

L'influence de la structure de l'industrie ou du marché sur la situation concurrentielle et les stratégies compétitives des entreprisesest discutée intensivement dans l'économie industrielle. La structure d'un secteur ou d'un marché est décrite comme un système interdépendant et dynamique de plusieurs variables comprenant, notamment, le degré de la concentration et le comportement concurrentiel des 


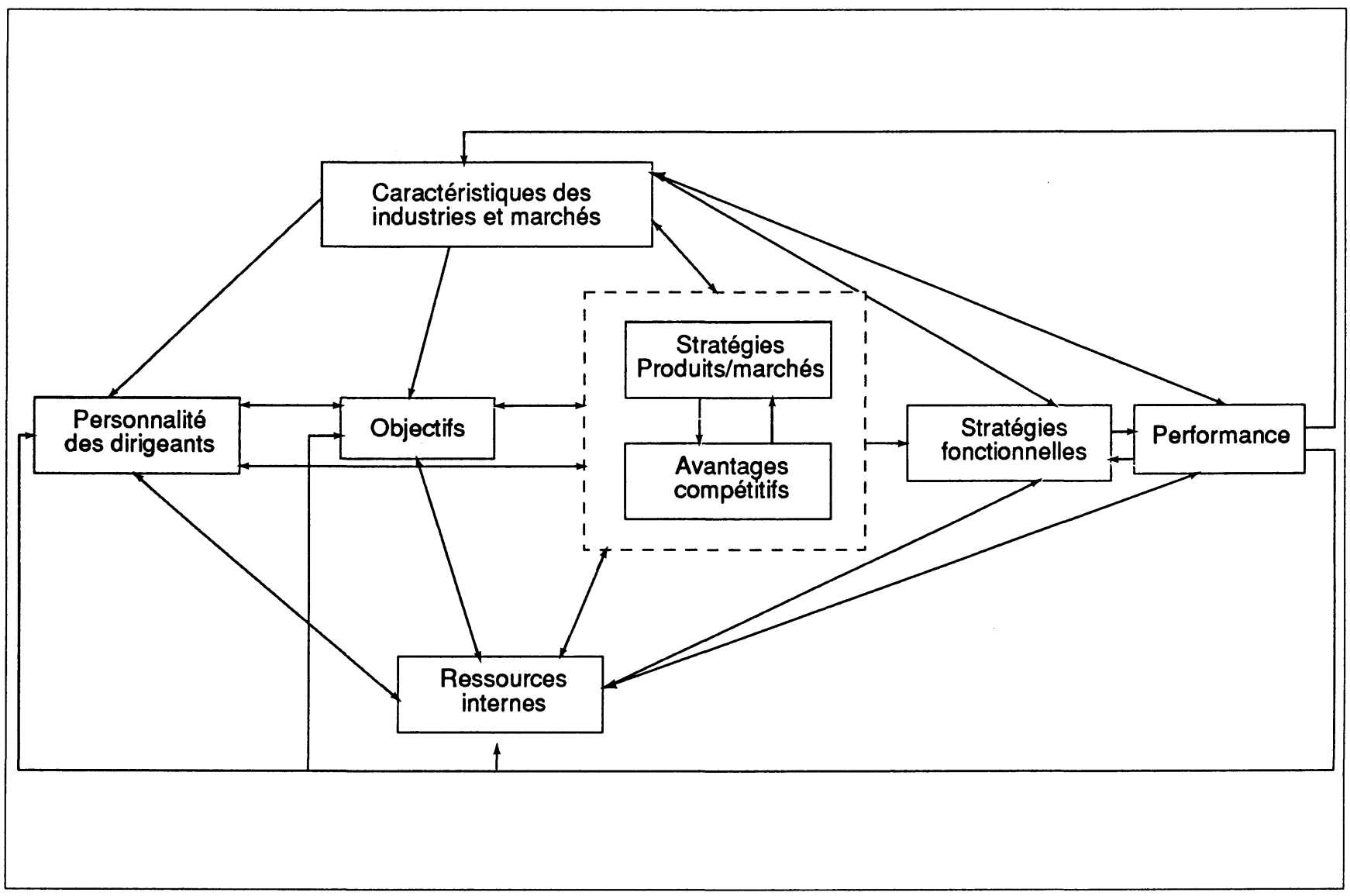

| 
entreprises existantes, les barrières d'entrée et de sortie, la menace de produits de substitution, le pouvoir de négociation des clientset des fournisseurset les interventions du gouvernement (Porter, 1980, Bamberger, 1985). Plusieurs de ces facteurs sont fortement liés aux technologies ainsiqu'aux effets d'économies d'échelle, d'économie de champs (économies of scope) et d'expériences.

Les caractéristiques de la demande et les conditions structurelles déterminent les critères de succès du marché, c'est-à-dire les ressources ou aptitudes dont l'entreprise doit disposer pour réussir sur un marché. Les critères de succès, les avantages compétitifs et les compétences distinctives devraient être distingués clairement. Les critères de succès constituent des caractéristiques, des exigences du marché. Par contre, les avantages compétitifs sont des avantages positionnels sur le marché qu'une entreprise développe par l'élaboration de certaines ressources (compétences distinctives) (figure 2).

Même si le développement des avantages compétitifs devrait correspondre aux conditions des marchés et à leurs critères de succès, il n'y a pas de relations déterministes. Dans beaucoup de branches, il existe une liberté d'action telle que les concurrents peuvent élaborer différents types d'avantages compétitifs. Dans une certaine branche, par exemple, certaines entreprises concurrencent sur la base d'une large gamme de produits, la qualité et une politique de marque tandis que d'autres fondent leur compétitivité sur une gamme étroite et des coûts et des prix bas. Cependant, dans d'autres branches il n'y a pas de degré de liberté : elles exigent, par exemple, nécessairement des coûts et des prix bas. Selon le nombre et la taille des avantages compétitifs possibles, différents environnements concurrentiels peuvent être distingués (Lochridge, 1981).

Si on accepte l'inexistence de relations déterministes entre les conditions de l'industrie ou du marché et les avantages compétitifs d'une entreprise, on devrait élargir l'analyse des déterminants par des facteurs supplémentaires.

\subsection{Les ressources internes}

Par conséquent, lesressources intemes del'entreprise peuventêtre considérées comme une deuxième classe de déterminants. Les rapports entre les ressources interneset les avantages compétitifs sont caractérisés par une interrelation dynamique : d'une part, les potentialités disponibles, par exemple sous forme d'équipements de production, de savoir faire technologique, de capacités de $R \& D$, de qualification de la main d'oeuvre, du système de distribution ou de la culture d'entreprise déterminent quel type d'avantage compétitif une entreprise peut ou devrait élaborer.

Il est un principe stratégique classique que la stratégie d'une entreprise devrait se fonder sur ses forces. D'autre part, la mise en oeuvre des avantages compétitifs, notamment par le biais des stratégies fonctionnelles, inclut le développementet l'adaptation de ressources spécifiques. Des avantages positionnels ont leur base, comme esquissé ci-dessus, dans le développement des compétences distinctives. 
Fig. 2 Les relations entre critères de succès, avantages compétitifs et ressources de l'entreprise

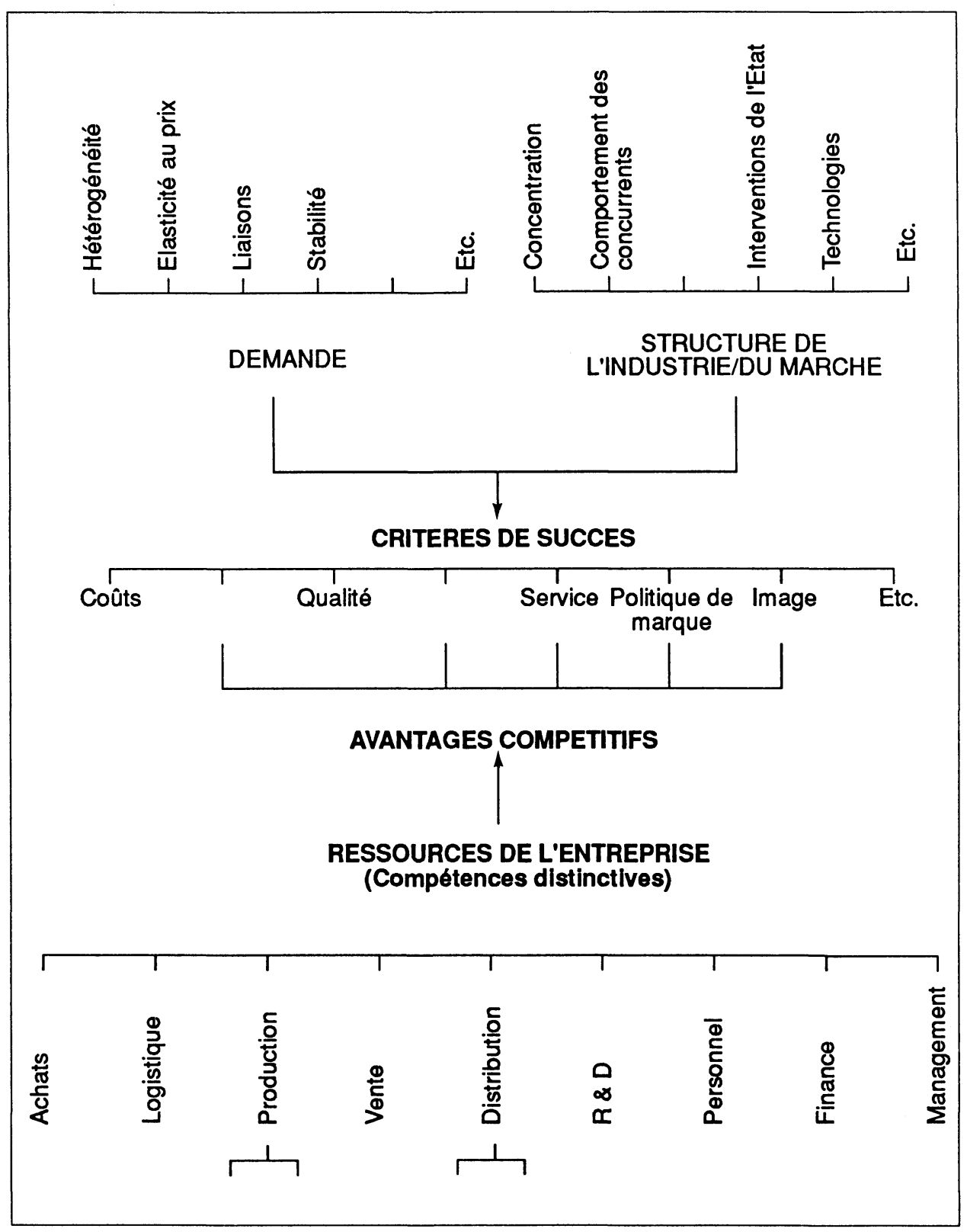




\subsection{Les objectifs de l'entreprise}

Les objectifs de l'entreprise, notament par rapportaux bénéfices, la croissance ou la part de marché, sont considérés comme un troisième type de déterminant. On peut supposer que, par exemple, des entreprises qui recherchent sur les marchés une forte croissance,élaborent d'autres types d'avantages compétitifs que des entreprises essayant de consolider leur position.

On peut supposer que les objectifs eux-mêmes sont influencés par la personnalité des décideurs stratégiques et reflètent la situation externe et interne ainsi que la performance actuelle et passée de l'entreprise. Enfin, on peut présumer que les relations entre les objectifs et les avantages compétitifs ne sont pas unilatérales. L'importance des sous-objectifs, tels que la productivité, la réduction des coûts, l'augmentation de la qualité, la flexibilité ou la créativité n'est pas indépendante du choix de certains avantages compétitifs.

\subsection{La personnalité des décideurs stratégiques}

C'est déjà, dans la théorie stratégique, presque une hypothèse classique que la personnalité des dirigeants et particulièrement leurs valeurs et attitudes, déterminent directement et indirectement le comportement stratégique de leurs entreprises (Bamberger, 1987a). On suppose que leurs valeurs économiques, sociales ou esthétiques, que leurs attitudes par rapport au changement, l'innovation, la croissance, la concurrence, le pouvoir, la tradition, l'internationalisation ou la participation influent sur les objectifs poursuivis, le choix des stratégies, mais également, par exemple, sur le style de direction ou la structure d'organisation. On peut supposer que l'influence des caractéristiques personnelles des dirigeants sur le comportement de leurs entreprises est beaucoup plus directe dans les entreprises de petite et moyenne taille que dans les grandes entreprises qui possèdent des structures plus complexes et souvent pluralistes.

Cette perspective, un peu volontariste, de l'importance de la personnalité des dirigeants pour le comportement stratégique en général et les stratégiescompétitives en particulier devrait être différenciée de plusieurs façons : les conditions externes et internes ne peuvent pas seulement limiter la liberté d'action et les alternatives, mais également influencer, par exemple, par des processus de socialisation, les traits personnels des managers. Ainsi, les stratégies compétitives poursuivies peuvent former certaines orientations ou exiger, dans le cadre de leur mise en oeuvre, le choix de certains types de dirigeants.

\subsection{Les stratégies produits/marchés}

Enfin, les choix par rapport aux produits et marchés, et du type d'avantages compétitifs sont fortementinterdépendants. La réussite d'une stratégie de spécialisation dépend de sa cohérence avec le type d'avantages compétitifs choisi. Ainsi, la 
définition des activités de l'entreprise ou d'un domaine d'activité stratégique par rapport aux produits, le type et le nombre de groupes de clients, et la détermination du type d'avantages compétitifs que l'entreprise veut élaborer, sont fortement interdépendants. Le nombre de groupes de produits et les liaisons entre les différents couples produits/marchés d'une entreprise peuvent posséder, par exemple, par des effets d'expériences partagées, une influence sur le développement des avantages compétitifs.

L'interaction des différents facteurs internes et externes, le comportement stratégique et sa mise en oeuvre déterminent la performance de l'entreprise qui ellemême peut avoir des effets de rétroaction sur ces différents éléments (figure 1).

\section{$3 \quad$ L'élaboration des avantages compétitifs dans les P.M.E.}

Le cadre de référence décrit, forme la base d'une étude empirique des facteurs que les entreprises de petite et moyenne taille utilisent pour développer des avantages compétitifs sur leurs marchés. Elle n'inclura pas toutes les variables et leurs relations, décrites en figure 1 . L'analyse se concentrera, à ce niveau, sur quelques relations fondamentales. L'étude est exploratoire.

\subsection{La méthodologie}

L'étude a été effectuée dans le cadre du projet de recherche international STRATOS («Strategic Orientations of Small Firms») 2 L'enquête fut réalisée en 1985 dans huit pays européens ${ }^{3}$.

L'échantillon se réfère à 1135 entreprises issues de trois branches : habillement, industrie agro-alimentaire et électronique. Les branches ontété choisies sur la base de plusieurs critères tels que: les biens de consommation et industriels, la dynamique, la phase d'évolution, etc...

2 Pour une description des bases théoriques et méthodologiques du projet voir Bamberger (1983), (1986 a), (1986 b).

3 Le groupe «STRATOS» se compose d' équipes des pays suivants : AUTRICHE : Erwin Froehlich, J. Hanns Pichler, Institut für Gewerbeforschung/Wien Wirt-schaftuniversität Wien BELGIQUE : Rik Donckels, Universitaire Faculteiten Sint Aloysius Brussel/KMOStudiecentrum ALLEMAGNE FEDERALE : Eduard Gabele, Universität Bamberg/Lehrstuhl für Betriebswirtschaftslehre FINLANDE : Antti J. Haahti, Allan Lehtimäki, Helsinki School of Economics, Tampere University of Technology/Institute of Industrial Economics FRANCE : Ingolf Bamberger, Université de Rennes I, Institut de Gestion de Rennes, Département Stratégie et Structure PAYS BAS : Cees Koning, Jan P.V.D. Wilde, Vrije Universiteit Amsterdam/Raad voor het midden-en Kleinbedrijf SUISSE : Klaus Haake, Hans J. Pleitner, Hochschule St Gallen f. Wirtschafts und Sozialwissenschaften/Schweizerisches Institut für gewerbliche Wirtschaft GRANDE BRETAGNE : Alastair Weir, Cliff Bohman, Humberside Business School, Hull. 
Les petites et moyennes entreprises ont été définies comme des entreprises ayant des effectifs de 1 à 500 salariés. Les entreprises devraient être indépendantes des groupes. La collecte des données fut effectuée par le biais des interviews administrés sous forme de questionnaire. Les interviewés étaient des membres du niveau le plus élevé de la structure d'organisation c'est-à-dire, en général, le dirigeant-propriétaire ou un dirigeant salarié, directeur général, gérant etc....

Pour connaître les bases des avantages compétitifs de leurs entreprises, nous avons posé la question suivante :

"Quel est le degré d'importance que vous attribuez aux facteurs suivants pour obtenir un avantage compétitif sur votre marché principal ?»

L'importance des 26 facteurs présentés fut mesurée sur une échelle de 5 points allant «d'aucune importance» (1) à une «très grande importance» (5). Les caractéristiques des marchés et des industries, les objectifs et les stratégies ont été mesurés par des variables avec différents types d'échelles. Le fait qu'elle n'ait pas mesuré les attributs des marchés et des industries directement, mais seulement par le biais de la perception des dirigeants, peut-être considéré comme une faiblesse de l'étude.

\subsection{Les résultats descriptifs}

Le tableau 1 décrit les résultats par rapport à l'importance relative des 26 facteurs pour l'ensemble des entreprises étudiées.

Si le pouvoir explicatif de ces résultats globaux est relativement faible, on peut néanmoins retenir, en tant que premier résultat, que la qualité du produit est considéré de loin comme le facteur le plus important, pour obtenir des avantages compétitifs sur les marchés. $61 \%$ des entreprises interviewées lui attribuent une importance «très grande», $32 \%$ une «importance grande». L'importance éminente de la qualité est confirmée par des résultats par rapport aux objectifs de l'entreprise et de l'entrepreneur.

En second lieu, des moyennes élevées et des écarts-types faibles de certains facteurs laissent supposer qu'il y a certains facteurs de base qui sont considérés par la plus grande majorité des entreprises comme des exigences fondamentales à leur compétitivité.

Une analyse factorielle a regroupé les 26 items en six facteurs globaux utilisés par les P.M.E. pour élaborer des avantages compétitifs sur leurs marchés. On peut les appeler :

- Compétence et réputation

- Potentialité de marketing

- Compétence technologique et service

- Conditions financières

- Créativité et différenciation des produits

- Coûts bas et politique des prix. 
Tab. 1 L'importance des facteurs (moyenne)

\begin{tabular}{|c|c|c|}
\hline Classement & Facteurs & $\begin{array}{l}\text { Importance } \\
\text { moyenne }\end{array}$ \\
\hline 1 & Qualité des produits & 4,53 \\
\hline 2 & Régularité de la livraison & 4,41 \\
\hline 3 & Renommée de l'entreprise & 4,30 \\
\hline 4 & Qualité de la main d'oeuvre & 4,22 \\
\hline 5 & Flexibilité de l'entreprise & 4,14 \\
\hline 6 & Qualité du management & 4,09 \\
\hline 7 & Bonne image locale et contacts personnels & 4,02 \\
\hline 8 & Capacité financière & 3,96 \\
\hline 9 & Les achats & 3,85 \\
\hline 10 & Climat social & 3,85 \\
\hline 11 & Coûts faibles & 3,81 \\
\hline 12 & Créativité & 3,80 \\
\hline 13 & Image de marque & 3,74 \\
\hline 14 & Ventes personnelles & 3,73 \\
\hline 15 & Conditions de paiement & 3,67 \\
\hline 16 & Politique de prix & 3,64 \\
\hline 17 & Techniques modernes de production & 3,57 \\
\hline 18 & Part de marché & 3,52 \\
\hline 19 & Formes et couleurs (design des produits) & 3,51 \\
\hline 20 & Capacité d'engineering & 3,45 \\
\hline 21 & Canaux de distribution & 3,43 \\
\hline 22 & Service après vente & 3,42 \\
\hline 23 & $\begin{array}{l}\text { Diversité des produits à l'intérieur des groupes } \\
\text { de produits }\end{array}$ & 3,31 \\
\hline 24 & Publicité / Promotion des ventes & 3,16 \\
\hline 25 & Assistance technique avant la livraison & 2,82 \\
\hline 26 & Taille de la force de vente & 2,73 \\
\hline
\end{tabular}

Le premier facteur "compétence et réputation» regroupe les items qui peuvent être considérés comme des facteurs (de réussite) de base, c'est-à-dire la qualité des produits, la renommée de l'entreprise, la régularité de la livraison, la qualité de la main d'oeuvre et du management ainsi que, dans un moindre degré, une bonne image locale et des contacts personnels. 
L'importance des cinq autres facteurs et de leurs éléments respectifs varie beaucoup plus en fonction des conditions externes et internes de l'entreprise. Les résultats de l'analyse factorielle seront utilisés pour l'étude des relations entre les avantages compétitifs élaborés et les autres variables. Cependant, les items initiaux seront utilisés dans le cas d'une nécessité d'analyse plus différenciée.

\subsection{Les avantages compétitifs et leurs liaisons avec des facteurs externes et Internes}

L'étude de ces relations se fera dans une première étape sous forme d'analyses bivariées. Elles sont basées, selon le niveau des échelles, sur des corrélations, des t-tests ou des analyses de variance. Un accent particulier sera mis sur les conditions des industries et marchés et les stratégies produits/marchés.

\subsubsection{Les conditions des industries et marchés}

Partant de l'hypothèse que l'élaboration des avantages compétitifs est fortement influencée par les conditions concurrentielles, il semble intéressant d'étudier la branche comme premier déterminant potentiel. La branche est pertinente dans la mesure où elle représente des conditions fondamentales de l'entreprise par rapport à la demande, des structures concurrentielles et les technologies.

Le tableau 2 montre les résultats par rapport aux trois branches électronique, habillement et agro-alimentaire. Les astérisques indiquent (sur la base d'une analyse des variances) des différences significatives dans l'importance que les entreprises, issues des trois branches précitées, attribuent aux différents facteurs.

Comme on le supposait, les entreprises des trois secteurs développent, mis à part des facteurs de base communs, différents types d'avantages compétitifs :

- Les entreprises de l'industrie électronique fondent des avantages compétitifs, de façon significative, plus sur la compétence technologique et le service (assistance technique avant la livraison, capacité d'engineering, service après vente). Cependant, elles essaient beaucoup moins d'obtenir des avantages compétitifs par des coûts bas et la politique de prix, les conditions financières, les achats et la régularité de la livraison que les entreprises de l'habillement et de l'industrie agro-alimentaire.

- Les résultats sont à compléter pour l'industrie de l' habillement, en comparaison avec les deux autres branches, par une plus forte importance des formes et des couleurs (design) de produits et de la créativité.

- La stratégie des entreprises agro-alimentaires se distingue de celles des deux autres branches de telle façon qu'elles basent leur compétitivité plus sur les canaux de distribution, la qualité des produits et une bonne image locale et des contacts personnels. Par ailleurs, elles attribuent moins d'importance à la créativité, le «design» des produits, la capacité d'engineering et la flexibilité. 
Tab. 2 La comparaison entre les trois branches

1 Politique des prix * $(<0,01)$

2 Les coûts faibles * $(<0,001)$

3 Diversité des produits à l'intérieur des groupes de produits

4 Qualité du produit * $(<0,01)$

5 Régularité de la livraison * $(<0,01)$

6 Techniques modernes de production

7 Assistance technique avant la livraison * $(<0,001)$

8 Service après-vente * $(<0,001)$

9 Formes et couleurs (design) du produit * $(<0,001)$

10 Bonne image locale et contacts personnels * $(<0,05)$

11 Qualité de la main d'oeuvre

12 Canaux de distribution * $(<0,001)$

13 Publicité/promotion des ventes

14 Vente personnelle

15 Taille de la force de vente

16 Créativité * $(<0,001)$

17 Capacité d'engineering * $(<0,001)$

18 Image de marque

19 Flexibilité de l'entreprise * $(<0,001)$

20 Renommée de l'entreprise

21 Qualité du management

22 Part de marché

23 Achats * $(<0,001)$

24 Conditions de paiement * $(<0,001)$

25 Capacité financière * $(<0,01)$

26 Climat social ${ }^{*}(<0,05)$

\begin{tabular}{|c|c|c|c|}
\hline 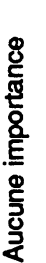 & 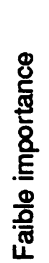 & 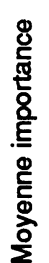 & 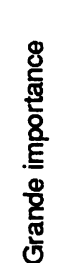 \\
\hline
\end{tabular}

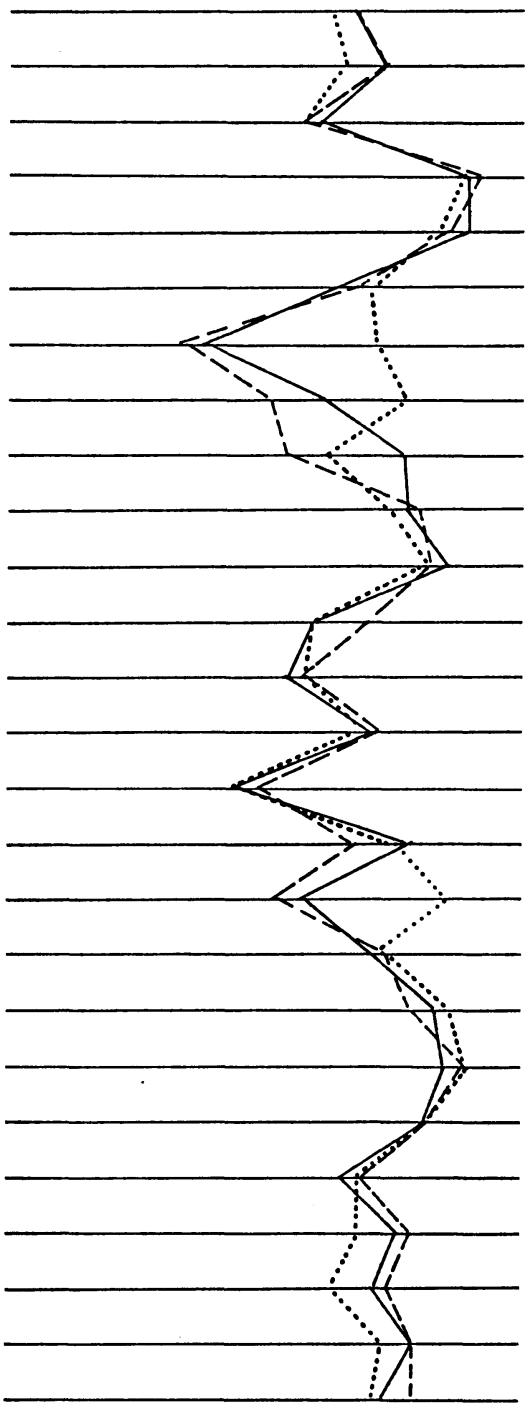

E H A 
Les caractéristiques communes et les différences entre les branches, mais également le fait que des différences considérables existent à l'intérieur des trois branches, indiquent que la branche ne constitue qu'un indicateur global pour les conditions concurrentielles des entreprises.

Ainsi, il est intéressant de savoir quelles relations existent, indépendamment de la branche, entre les caractéristiques de la demande, de la structure du marché ou de la technologie et les avantages compétitifs élaborés.

A titre d'exemple, les résultats par rapport à la sensibilité de la demande au prix, l'homogénéité et la stabilité de la demande seront décrits :

- Si la sensibilité de la demande au prix est élevée, les entreprises fondent leur compétitivité plus sur les coûts bas et la politique de prix, les techniques modemes de production, les achats et la capacité financière. Cependant, il y a une relation négative entre la sensibilité de la demande au prix et l'importance des services avant et après la vente et la qualité de la main-d'oeuvre.

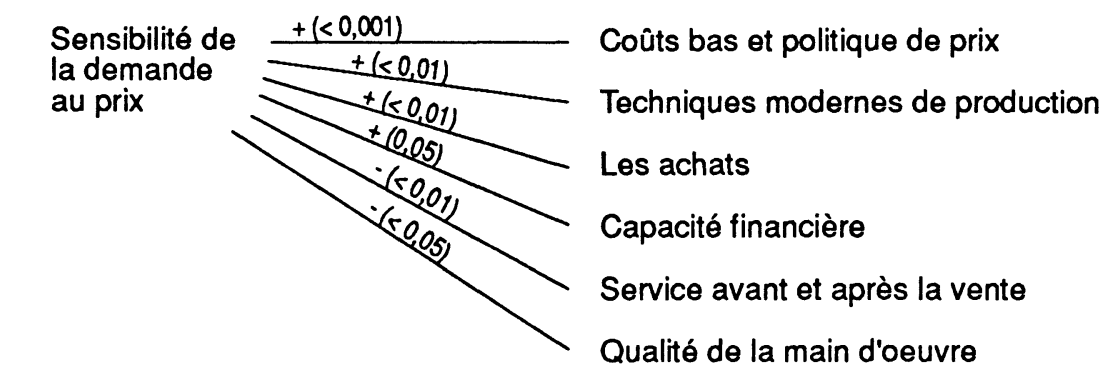

- Les entreprises qui se voient confrontées à une demande hétérogène et qui, de cette façon, offrent des produits non standardisés, mettent plus l'accent sur la créativité et la différenciation des produits, la compétence technologique et le

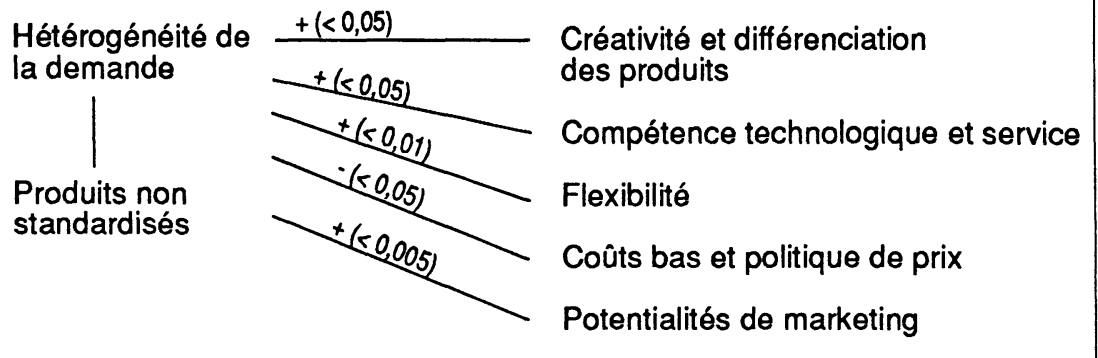


service ainsi que la flexibilité, que des firmes avec une demande homogène et des produits standardisés. Par contre, les entreprises avec une demande homogène et des produits standardisés développent plus d'avantages compétitifs sur la base des coûts bas et la politique de prix et les potentialités de marketing.

- La stabilité de la demande est notamment liée à l'importance mise sur les potentialités de marketing, les formes et les couleurs (design) des produits, la compétence technologique et le service.

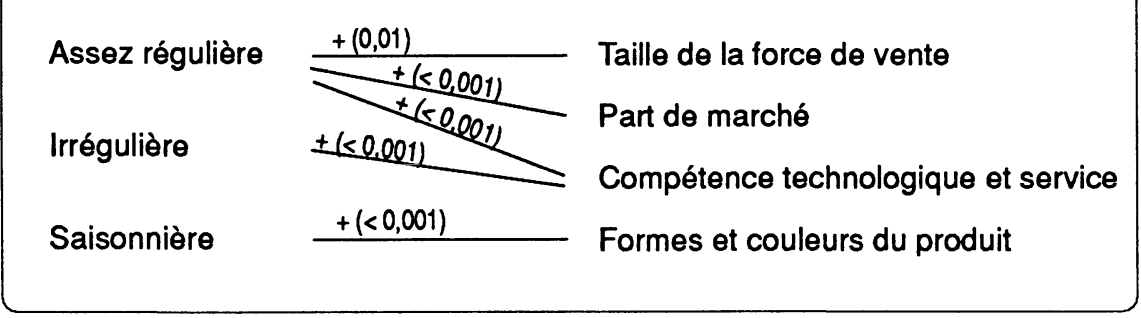

\subsubsection{Les stratégies produits/marchés}

Les relations entre l'homogénéité de la demande et la standardisation des produits signalent une forte interdépendance entre les caractéristiques de l'industrie ou du marché, les stratégies produits et marchés et le type des avantages compétitifs que l'entreprise développe.

L'hypothèse des relations entre les stratégies produits/marchés et lesavantages compétitifs est également confirmée par des résultats (d'analyses bivariées) par rapport aux nombres de groupes de produits, l'extension géographique des marchés, le type et le nombre de clients ainsi qu'au comportement stratégique des soustraitants.

- Par exemple, il y a une relation positive entre le nombre de groupes de produits d'une entreprise (et d'ailleurs, du nombre des clients ) et l'importance attribuée à l'élaboration des potentialités de marketing, mais également aux coûts bas, à la politique de prix et aux conditions financières.

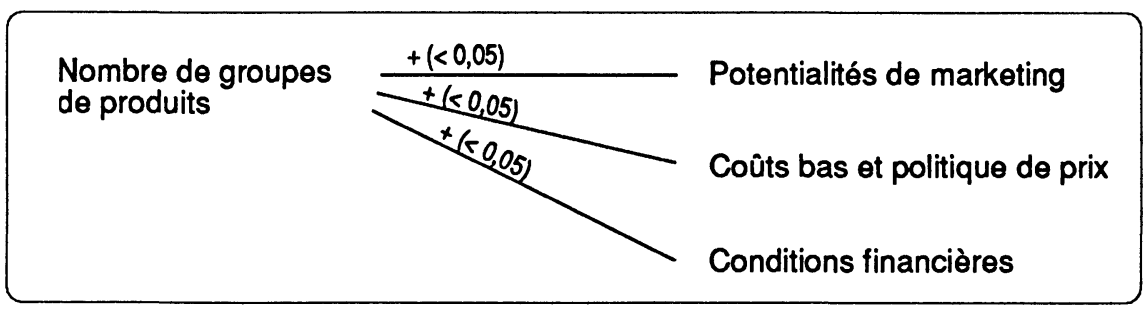


- Des résultats fort intéressants sont à constater par rapport au développement des avantages compétitifs en fonction du type de clients (consommateurs finaux, distributeurs, entreprises industrielles, Etatet collectivités publiques etentreprises de service) et de l'extension géographique des marchés (marchés locaux, régionaux, nationaux, Europe de l'Ouest et de l'Est, marchés mondiaux).

L'extension géographique du marché, par exemple, est liée à l'importance qui est accordée à la compétence technologique et au service, la créativité et le design des produits, la flexibilité, à la distribution et la publicité, mais aussi à l'image et la renommée de l'entreprise. Il est particulièrement intéressant de constater que les marchés de l'Est semblent exiger des compétences particulières. C'est apparemment aussi le cas de l'Etat et des collectivités publiques en tant que groupe de clients.

- Des entreprises qui agissent en tant que sous-traitants (preneurs d'ordres) essaient d'obtenir des avantages compétitifs davantage par l'élaboration de la compétence technologique et du service que par le biais des potentialités de marketing, la créativité et la différenciation des produits et les achats que des entreprises qui n'ont pas d'activités de sous-traitance.

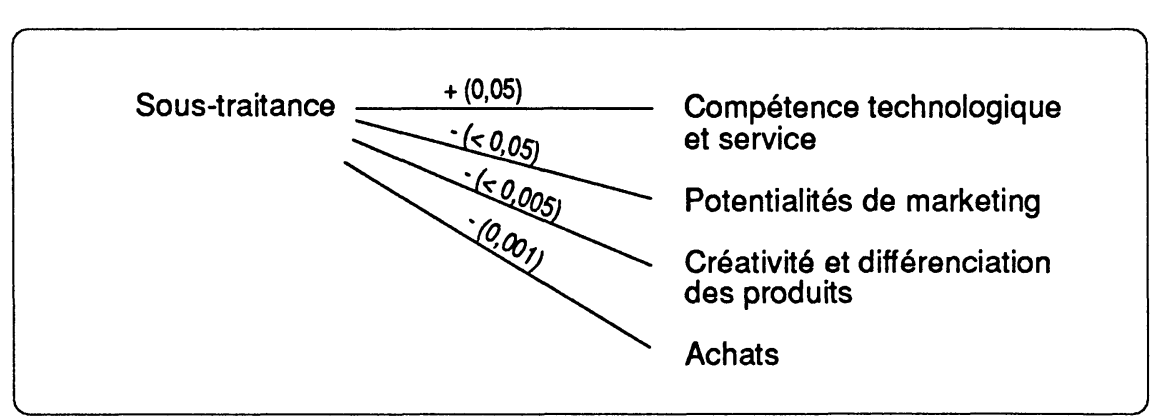

Contrairement à ce que l'on pouvait supposer, il n'y a pas de différences significatives par rapport à l'importance des coûts bas et de la politique de prix ou de la flexibilité.

\subsubsection{Autres facteurs}

D'autres analyses révèlent des liaisons entre d'autres caractéristiques de l'entreprise, comme sa taille, sa technologie (par exemple intensité capitalistique) et, notamment, ses objectifs d'une part et l'élaboration de certains types d'avantages compétitifs d'autre part. Ainsi, l'importance des objectifs, comme la croissance, les parts de marché, productivité et réduction des coûts, indépendance ou créativité, est fortement liée au développement d'une combinaison spécifique de facteurs. 


\section{$4 \quad$ L'élargissement de l'étude}

Les résultats des analyses bivariées semblent confirmer les hypothèses de base par rapport aux relations entre les conditions concurrentielles, les stratégies et objectifs des entreprises et les facteurs qu'elles utilisent pour développer des avantages compétitifs.

Cependant, cesanalyses présentent notamment trois faiblesses ou limitations :

- Le cadre de référence développé,ainsi que les résultats empiriques, signalent une interdépendance des conditions de marché, des stratégies produits/marchés, des ressourcesinternes, des avantages compétitifs et de la performance de l'entreprise. On ne peut la saisir qu'insuffisamment avec des méthodes bivariées. Pour cette raison des interprétations causales des résultats ont été évitées.

- Les relations entre les différentes variables ont été étudiées, compte tenu du grand nombre de variables de l'enquête et de l'hétérogénéité de l'échantilon, à un niveau relativement global et peuvent, de cette manière, cacher, en fonction des constellations, des relations plus spécifiques et, peut-être différentes.

- Certains complexes de variables, comme les caractéritiques de la personnalité des dirigeants, les conditions de la mise en oeuvre et la performance de l'entreprise, n'ont pas encore été introduites dans l'analyse empirique.

Les faiblesses décrites seront compensées par un élargissement de l'étude fondée sur des analyses multivariées. On essayera notamment d'identifier, en fonction des conditions concurrentielles et des orientations stratégiques, différents types d'entreprises.

\section{BIBLIOGRAPHIE}

BAMBERGER, I., Les valeurs des dirigeants de P.M.E. et leur influence sur le comportement stratégique et la performance de leurs entreprises, in : Sciences de Gestion, 1983, p. 1353 et sv.

BAMBERGER, I., Du cycle de vie des produits à l'évolution des secteurs ?, Cahiers Stratégie et Organisation, $\mathrm{n}^{\circ} 13 \mathrm{~b}$, Rennes, 1985.

BAMBERGER, I., Values and Strategic Behavior, in : Management International Review, $1986, \mathrm{n}^{\circ} 4$.

BAMBERGER, I., The STRATOS Project, Cahiers Stratégie et Organisation, n 18 , Rennes, 1986.

DAY, S.G. , Strategic Market Planning : The Pursuit of Competitive Advantage, St Paul etc., 1984. 
HENDERSON, B.D., The Anatomy of Competition, in : Journal of Marketing, 1983, spring, pp. 7-11.

HOFER, C.W. \& SCHENDEL, D., Strategy Formulation : Analytical Concepts, St Paul etc., 1978.

LOCHRIDGE, R.K., Strategies in the Eighties, Boston Consulting Group, 1981.

PORTER, M.E., Competitive Strategy, New York-London 1980.

PORTER, M.E., Competitive Advantage, New York-London 1985.

PUMPIN, C., Management strategischer Erfogspositionen, Bern und Stuttgart, 1983.

ROTHSCHILD, W.E., How to Gain (and Maintain) the Competitive Advantage in Business, New York etc., 1984. 